\title{
Comparative study of PFN antirotation vs bipolar hemiarthroplasty in unstable senile intertrochanteric fractures
}

\author{
Hrushikesh Saraf ${ }^{1}$, Shreepal Munot ${ }^{2, *}$ \\ Orthopaedic Surgeon, Dept. of Orthopaedics, Shashwat Hospital, Pune, Maharashtra, India
}

*Corresponding Author: Shreepal Munot

Email: drshreepalmunot@gmail.com

\begin{abstract}
Introduction: Treatment of intertrochanteric fracture in elderly is not only to mobilise early but also to avoid complications and morbidity. With available treatments it is difficult to address all the issue with same surgical option. A recent advance for treatment of senile unstable intertrochanteric fracture is PFN antirotation which may help in early mobilisation with less complications. The purpose of this study is to compare the outcome of PFNA and hemiarthoplasty and to study advantages, disadvantages, complications of both the groups.

Materials and Methods: 20 patients each were included in PFNA group and hemiarthroplasty group from January 2016 to January 2017 who satisfied the inclusion and exclusion criteria. Patient followed up upto 1 year. Patients were evaluated with multiple variables, complications and harris hip score.

Results: PFNA groups had significantly less blood loss, less surgery time, less hospital stay as compared to hemiarthroplasty group. Both groups had similar medical and orthopaedic complications. PFNA patients can be mobilised early as with hemiarthroplasty group. Hemiarthoplasty groups have better harris hip score in initial 3 months but similar score at the end of 1 year.

Conclusion: PFNA provide less morbidity to patients with early mobilisations and good functional outcome. It can definitely be a better option than replacement in elderly intertrochanteric fracture.
\end{abstract}

Keywords: PFNA, Bipolar, Intertrochanteric fracture, Hemiarthroplasty, Harris hip score, Replacement.

\section{Introduction}

Hip fractures are among the commonest injuries sustained by the elderly, predominantly over 60 years of age. Among the hip fractures $45 \%$ are intertrochanteric fractures and again $35 \%-40 \%$ of these fractures are unstable fractures. ${ }^{1}$

As the life expectancy is increasing, number of patients with postmenopausal or senile osteoporosis are increasing proportionately. So there is increase in demand for better treatment of proximal femoral fractures. ${ }^{2}$

The primary treatment for intertrochanteric fractures is internal fixation or osteosynthesis by dynamic hip screws, intramedullary implants but osteosynthesis is always a challenge in osteoporotic patients due to complications like femoral head perforation, metal failure and varus collapse. ${ }^{3,4}$ In addition to intra-medullary devices for the treatment of intertrochanteric fractures, hemiarthroplasty is considered but not accepted as a primary treatment. ${ }^{5}$ Although hemiarthroplasty could shorten weight bearing and decrease implant related complications. ${ }^{6,7}$

Proximal femoral nail antirotation devices have been introduced recently to obtain better fixation in the presence of osteoporosis. These implant consists of intramedullary nail with proximal angulation of $6^{\circ}$. It provides rotational stability by compaction of cancellous bone with the helical blade into the femoral head. It is biomechanically proved that the helical blade has significantly higher cut out resistance than other commonly used screw systems. So PFNA blade appears to be biomechanically suitable implant for unstable trochanteric fractures and trochanteric fractures associated with osteoporotic bones. ${ }^{8}$

The purpose of present study is to compare the outcome of PFN antirotation with bipolar hemiarthroplasty in reference to advantages, disadvantages, complications.

\section{Materials and Methods}

20 patients each were included in PFNA group and hemiarthroplasty group from January 2016 to January 2017 who fulfil the inclusion criteria.

Inclusion Criteria: 1) Age > 70 years, 2) Unstable intertrochanteric fractures 3) Patient mobile before the fracture. 4) Medically fit for surgery.

Exclusion Criteria: 2) Age $<70$ years, 2) Bedridden patient before fracture 3) Pathological fractures 4) Polytrauma 5) Medically unfit for surgery.

All the patients were admitted in emergency department of our hospital. Thomas splint was put for initial immobilisation and to reduce pain. Preoperative blood test was sent to pathology laboratory. Physician opinion taken regarding fitness for surgery. A written, informed consent taken explaining details of surgery, risk factors, postoperative rehabilitation, medical and surgical complications. Pre anaesthetic check up was done a day prior to surgery. ASA grading recorded.

Surgery steps -1 ) PFNA

After induction of anaesthesia patient put on lateral decubitus position on radiolucent table. Other leg maximally flexed and pillow kept in between the legs. Fracture reduction confirmed under image intensifier in AP view and lateral view (20 degree oblique to avoid 
overlap with other hip). After closed reduction, an about $5 \mathrm{~cm}$ longitudinal incision was made approaching the greater trochanter. Entry taken with awl through tip of GT. Guide pin passed through the tip into the medullary canal. Nail passed over the guide pin without reaming. Helical blade put centrally/inferiorly in anteroposterior view and centrally in lateral view. Distal static bolt put. (Fig. 1) wound closed in layers without drain.

2) Bipolar hemiarthroplasty

After induction of anaesthesia patient put on lateral decubitus position. Moore's approach for all the cases. $10 \mathrm{~cm}$ curved incision centering over GT. Short external rotators ligated and cut. Removal of head in intertrochanteric fracture is difficult. Head extractor is put and capsule is released all around. Other option is to cut it subcapitally with saw as in neck femur fracture and then remove remaining neck with piecemeal. Fracture pattern is assessed. Usually LT is reconstructed first to get a anatomical landmark using SS wire. Then Canal is reamed and serially broached. Trial stem put. Final stem is put with/without cement. Final head put after trial reduction. Greater trochanter then fixed with $\mathrm{k}$ wires /encirclage/tension band wiring depending on fracture pattern. (Fig. 2) Wound closed over negative suction drain.

Post operatively abduction pillow kept for hemiarthroplasty patient. Haemoglobin checked next morning and accordingly blood transfused if required. Most of hemiarthroplasty patients started full weight bearing walking with walker support from post op day one.

PFNA patients also started full weight bearing walking with walker from post op day one. All patients were given DVT prophylaxis like factor Xa inhibitor/ direct thrombin inhibitor/ clopidogrel/aspirin etc. till 3 weeks.

All patient planned to discharge usually post op day 2-3.Criteria to discharge 1) Minimum 4 dose of iv antibiotics, 2) Dry wound 3) Drain should be removed in case of replacement 3) should have commode training 4) General condition should be satisfactory.

Patient called for follow up at 1 month, 3 month, 6 month, 1 year. X-rays taken at each follow up and Harris hip score calculated.

Patients of PFNA group were gradually mobilised from walker support to stick support to walking without support. Hemiarthroplasty group mobilised with walker for one week and then to stick support /without support depending upon general condition of patient.

Data statistically analysed using fisher's exact test for categorical variables and student's $\mathrm{T}$ test for continuous variables. Two tailed $\mathrm{P}$ value of less than 0.05 considered to be significant.
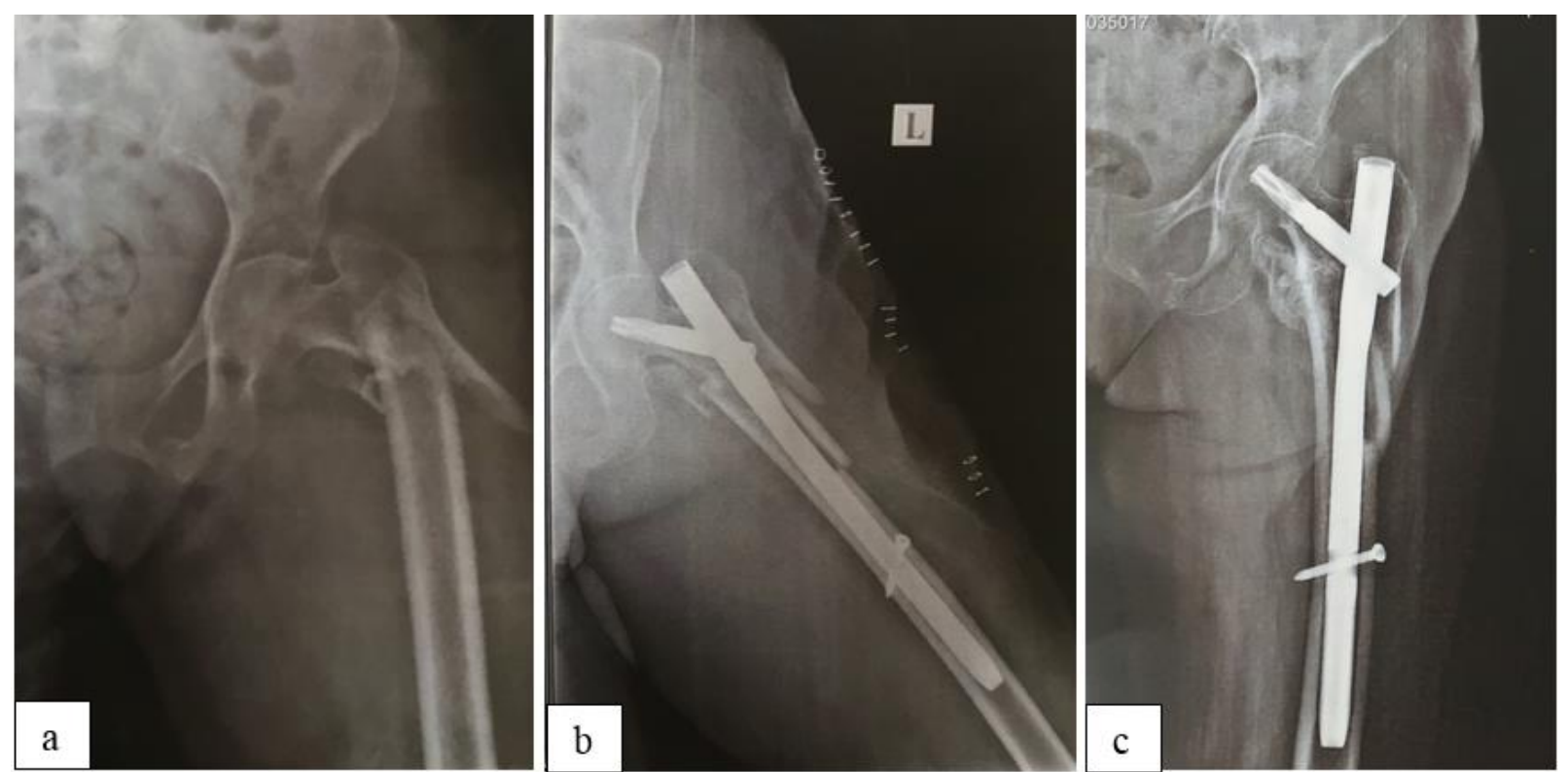

Fig. 1 A) Pre op x-ray unstable intertrochanteric fracture b) immediate post op x-ray -helical blade put centero-inferiorly c) union at 4 month post op 


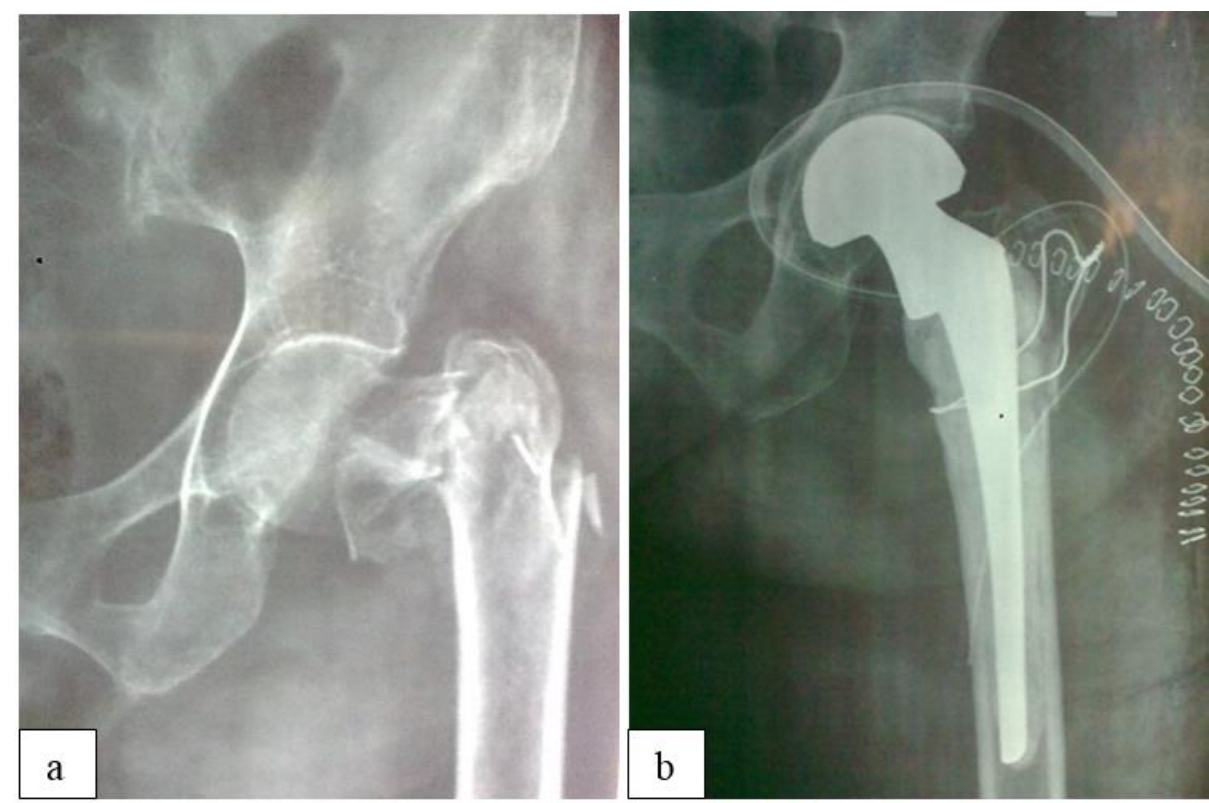

Fig. 2 a: Pre op x-ray -comminuted intertrochanteric fracture; b: Post op x-ray -GT, LT fixed with encircalge wire

\section{Results}

In both groups 20 patients were included and their data analysed. Baseline characteristics like age, sex, side, type of fracture, ASA grading, comorbid conditions were recorded. There were no significant difference in above characteristics $(\mathrm{P}>0.05)$ (Table 1)

In hemiarthroplasty group 15 patients underwent cemented bipolar replacement, 5 patients underwent uncemented bipolar replacement.

PFNA group scored significantly better with respect to intraoperative blood loss, surgical time, hospital stay compared to hemiarthroplasty group. $(\mathrm{P}<0.05)$ (Table 2)

There was less intraoperative complications in PFNA group as compared to hemiarthroplasty. $(\mathrm{P}=0.047)$. (Table 2).In hemiarthroplasty group three patients had hypotension with bradycardia following cementing. Two patients had symptomatic fat embolism with hypotension, desaturation and tachypnea. All patients were immediately resuscitated and shifted to ICU for further management. In PFNA group none of the patients had intraoperative complications.

Hemiarthroplasty group definitely had better functional outcome using Harris hip score till first three months $(\mathrm{P}<0.05)$ as compared to PFNA group. But both groups had similar score at 6 month and 1 year $(\mathrm{P}>0.05)($ Table 3$)$

There was significant difference in the incidence of wound complications in both groups $(0 \%$ vs $25 \%, \mathrm{P}=$ 0.047)(Table 4). In PFNA group none of the patients had wound infection or post operative wound drainage following DVT Prophylaxis. In Hemiarthroplasty group two patients have superficial wound infection. Wound debridement and through lavage done in both cases and antibiotics was given as per culture and sensitivity for 6 weeks. Both patients recovered following the treatment. Three patients had postoperative wound drainage following oral anticoagulants. Regular dressing was done and anticoagulants were stopped. There was no significant difference in implant related orthopaedic complications $(10 \%$ vs $15 \%, \mathrm{P}>0.999)$ in both the groups. IN PFNA group one patient had screw cut out superiorly and varus collapse for which he was advised reoperation but patient was not willing for second surgery. Another patient had periprosthetic fracture (Vancouver type B1) following history of fall from bed. She was operated again and ORIF with DCP and screws done. In hemiarthroplasty group one patient had dislocation with breakage of tension band wire over GT. This patient was re-operated with open reduction and revision of TBW. One patient had breakage of tension band wiring over GT following history of fall. She was managed conservatively and advised non weight bearing walking with walker till GT union. One patient had migration of $\mathrm{k}$ wire medially noticed in one year follow up x-ray. As this patient didn't have any complaints she was counselled and monitored. There was no significant difference in reoperation rate in both groups $(\mathrm{P}=0.605)($ Table 4$)$.

Patient treated with Hemiarthroplasty had a trend of higher medical complications as compared to PFNA group. However results are not significant (5\% vs $20 \%$ $\mathrm{P}=0.342)$ (Table 4). Common causes includes pneumonia, hyponatremia. 
Table 1

\begin{tabular}{|l|c|c|c|}
\hline \multicolumn{1}{|c|}{ Characteristics } & PFN antirotation & Hemiarthroplasty & P value \\
\hline Age(years) & $82.4 \pm 3.9$ & $80.8 \pm 4.3$ & 0.230 \\
\hline Sex(M:F) & $10: 10$ & $9: 11$ & 0.999 \\
\hline Side (R:L) & $11: 9$ & $6: 14$ & 0.200 \\
\hline Boyd and griffin classification & & & 0.758 \\
\hline type II & $14(70 \%)$ & $12(60 \%)$ & \\
\hline type III & $2(10 \%)$ & $2(10 \%)$ & \\
\hline type IV & $4(20 \%)$ & $6(30 \%)$ & \\
\hline ASA Grade & & & 0.584 \\
\hline I & $2(10 \%)$ & $2(10 \%)$ & \\
\hline II & $9(45 \%)$ & $5(25 \%)$ & \\
\hline III & $8(40 \%)$ & $12(60 \%)$ & \\
\hline IV & $1(5 \%)$ & $1(5 \%)$ & \\
\hline Comorbidities & & & \\
\hline DM & $7(35 \%)$ & $5(25 \%)$ & 0.731 \\
\hline Hypertension & $6(30 \%)$ & $9(45 \%)$ & 0.515 \\
\hline Cardiac illness & $4(20 \%)$ & $6(30 \%)$ & 0.716 \\
\hline Asthma/COPD & $3(15 \%)$ & $3(15 \%)$ & 0.999 \\
\hline
\end{tabular}

M-male, F-female, R-right, L-left, DM-diabetes mellitus, COPD- chronic obstructive pulmonary disease

Table 2

\begin{tabular}{|l|c|c|c|}
\hline \multicolumn{1}{|c|}{ Variable } & PFN antirotation & Hemiarthroplasty & P values \\
\hline Surgical time (mins) & $40 \pm 6.09$ & $55 \pm 9.50$ & $<0.001$ \\
\hline intraoperative blood loss(ml) & $120 \pm 29.4$ & $300 \pm 49.4$ & $<0.001$ \\
\hline Intraoperative complications & $0(0 \%)$ & $5(25 \%)$ & 0.047 \\
\hline ICU requirement post op & $3(15 \%)$ & $8(40 \%)$ & 0.155 \\
\hline Hospital stay (days) & $4 \pm 1.19$ & $6 \pm 2.13$ & $<0.001$ \\
\hline Time to complete weight bearing & Immediate & Immediate & \\
\hline
\end{tabular}

Table 3

\begin{tabular}{|l|c|c|c|}
\hline \multicolumn{1}{|c|}{ Variable } & PFN antirotation & Hemiarthroplasty & P value \\
\hline Harris hip score at 1 month & $52.1 \pm 2.05$ & $61.4 \pm 3.75$ & $<0.001$ \\
\hline Harris hip score at 3 month & $64.05 \pm 2.61$ & $70.20 \pm 2.14$ & $<0.001$ \\
\hline Harris hip score at 6 month & $79.65 \pm 1.5$ & $79.95 \pm 1.82$ & 0.573 \\
\hline Harris hip score at 1 year & $84.35 \pm 2.58$ & $85.9 \pm 2.31$ & 0.053 \\
\hline
\end{tabular}

Table 4

\begin{tabular}{|l|c|c|c|}
\hline \multicolumn{1}{|c|}{ Variables } & PFN antirotation & Hemiarthroplasty & P value \\
\hline $\begin{array}{l}\text { Minor orthopaedic complications (wound } \\
\text { infection/excessive bleeding) }\end{array}$ & $0(0 \%)$ & $5(25 \%)$ & 0.047 \\
\hline $\begin{array}{l}\text { Major orthopaedic complications(implant } \\
\text { failure, dislocations, periprosthetic } \\
\text { fracture) }\end{array}$ & $2(10 \%)$ & $3(15 \%)$ & $>0.999$ \\
\hline Medical complications & $1(5 \%)$ & $4(20 \%)$ & 0.342 \\
\hline Reoperation rate & $1(5 \%)$ & $3(15 \%)$ & 0.605 \\
\hline
\end{tabular}

\section{Discussion}

Treating a senile intertrochanteric fracture poses a great challenge to orthopaedic surgeon especially when multiple options are available from conservative to internal fixation to replacement surgeries.

Internal fixation with PFN has advantage of small incision, less invasive, less tissue handling, less hospital stay, less operative time. ${ }^{9-11}$ Our study well supported the above advantages where PFNA group showed significant less blood loss $(100 \mathrm{ml}$ vs $300 \mathrm{ml}$, P
$<0.001$ ), less operative time (40 mins vs 55 mins, $\mathrm{P}$ $<0.001$ ), less hospital time (4 days vs 6 days, $\mathrm{P}<0.001$ ).

Hemiarthroplasty group had more wound complications $(25 \%)$ including wound complications secondary to DVT prophylaxis as compared to PFNA group (0\%). This results are comparable with study by Görmeli ${ }^{12}$ et al. Anticoagulants need to be stopped in those patients till wound heals which lead to cardiac risk in elderly patients.

Studies showed that internal fixation have disadvantage of screw cut out, periprosthetic fracture, 
head fracture, higher reoperation rate ${ }^{13,14}$ but our study contradicts and showed no significant difference in implant related and major orthopaedic complications ( $\mathrm{P}$ $>0.999$ ). Possible explanation can be 1) Helical blade results in increased surface contact between devices and femoral head cancellous bone, compressing rather than removing the limited amount of bone. 2) The tip of PFNA nail is especially designed to reduce the concentration of stress. ${ }^{8}$ In another study by R.K. simmermacker et $\mathrm{al}^{15}$ concluded PFNA to be effective method in treating unstable senile intertrochanteric fracture with low rate of internal fixation failure and related re-operation. E.soucanye et $\mathrm{a}^{16}$ showed similar results. Although there are many studies which showed lesser orthopaedic complications with hemiarthroplasty as compared to $\mathrm{PFN}^{6,17-20}$ but the complications associated with replacement like dislocations, cement related complications, fat embolism mainly depends on skill and experience of operating surgeon. Hemiarthroplasty in senile comminuted intertrochanteric fracture is far more difficult compared to hemiarthroplasty in neck femur fracture which require longer learning curve and surgeons expertise to identify and reconstruct anatomical landmark correctly. ${ }^{21}$ In our study even after taking all the precautions $15 \%$ of patient had cement related complication like hypotension, bradycardia and immediate intervention was required to resuscitate the patients. This complication can easily be avoided with PFN. In hemiarthroplasty broaching and reaming of medullary canal is necessary irrespective of cementation with leads to fat embolism in many patients as seen in $10 \%$ of our patients in hemiarthroplasty group. As unreamed PFNA was done in all of our cases, no incidence of fat embolism was noticed in PFNA group.

One unbiased advantage of hemiarthroplasty is early mobilisation which reduces the chances of medical complications like pulmonary complications, bed sores, DVT. ${ }^{22}$ In our study we mobilised PFNA patients on post operative day 1or 2 because of 1) added advantage associated with PFNA helical blade as described earlier 2) PFNA is minimal invasive surgery with minimal tissue handling, minimal blood loss which lead to less disturbance to normal physiology of body and early recovery to normal physiology 3)Operating surgeon took additional precaution to put helical blade centrally or inferiorly in AP view and centrally in lateral view. We documented less medical complications in PFNA group although insignificant $(\mathrm{P}=0.342)$.

R. Siwach et al, ${ }^{23} \mathrm{~N}$. Ozkayn et $\mathrm{al}^{24}$ showed better functional outcome of hemiarthroplasty group as compared to PFN group. Though our study showed hemiarthroplasty patients outscoring PFNA patients significantly in first 3 months Harris hip scores $(\mathrm{P}<0.001)$ but both groups showed similar Harris hip score at 6 month and 1 year $(\mathrm{P}>0.05)$. As the fracture unites in PFNA group, pain reduces and activity level increases which usually takes 4-5 months. PFNA preserve the natural hip, patients have no restriction in activities especially in Indian population where squatting and sitting cross leg is required for daily living and for religious activities.

\section{Conclusion}

PFNA definitely provide advantage for senile intertrochanteric fracture being less invasive, quick surgery, and less disruptive to normal physiology. It allows early weight bearing similar to hemiarthroplasty which has more risk of cementation, hypotension, wound related complications. PFNA being osteosynthesis procedure provide normal hip with no restriction of activities and similar functional outcome at the end of 1 year.

\section{Limitation}

Small sample size can alter the study results. Prefracture activity level not included in study which can affect the post-operative functional outcome. Also to study orthopaedic complications long term follow up is required.

\section{Conflict of Interest: None.}

\section{References}

1. Naushad Hussain, Hiren B. Patel, Nirmal D. Patil. Management of complex intertrochanteric fractures of the femur in elderly patients - dynamic hip screws or proximal femoral nails or arthroplasty. Int J Res Orthop. 2017;3(4):656-660.

2. Giannotti S, Bottai V, Pini E, Dell'osso G, De Paola G, Guido G. Clinical and surgical approach of severe bone fragility fracture: Clinical case of 4 fragility fracture in patient with heavy osteoporosis. Clin Cases Miner Bone Metab. 2013;10:52-55.

3. A. Enocson, L. Mattisson, C. Ottosson, L.J. Lapidus, Hip arthroplasty after failed fixation of trochanteric and sub trochanteric fractures: a cohort study with5-11 year follow-up of 88 consecutive patients, Acta Orthop. 83 (2012)493-498

4. C. D' Arrigo, D. Perugia, A. Carcangiu, E. Monaco, A. Speranza, A. Ferretti, Hiparthroplasty for failed treatment of proximal femoral fractures. Int Orthop. 2010;34:939_ 942.

5. Tang P, Hu F, Shen J, Zhang L, Zhang L.Proximal femoral nail antirotation versus hemiarthroplasty: a study for the treatment of intertrochanteric fractures. Injury. 2012;43:876-881.

6. S.G. Fichman, T.J. Mäkinen, O. Safir, A. Vincent, B. Lozano, A. Kashigar, et al., Arthroplasty for unstable pertrochanteric hip fractures may offer a lowerreoperation rate as compared to cephalomedullary nailing. Int Orthop. 2016;40:15-20.

7. B. Shen, J. Jiao, W. Kan, Comparison between artificial hip replacement andinternal fixation for treatment of intertrochanteric fractures in the elderly: a Meta-Analysis, Acta Med Univ Sci Technol Huazhong. 2014;43:586-594. 
8. WL Loo, M Med Orth, SYJ Loh, FRCS (Edin), HC Lee, FRCS (Edin). Review of Proximal Nail Antirotation (PFNA) and PFNA-2 -Our Local Experience. Malaysian Orthop J. 2011:5(2).

9. Parker MJ, Khan RJ, Crawford J, Pryor GA. Hemiarthroplasty versus internal fixation for displaced intracapsularhip fractures in the elderly. A randomised trial of 455 patients. J Bone Joint Surg Br. 2002;84:11501155.

10. Parker MJ, Pryor GA. Internal fixation or arthroplasty for displaced cervical hip fractures in the elderly: a randomised controlled trial of 208 patients. Acta Orthop Scand. 2000;71:440-446.

11. Puolakka TJ, Laine HJ, Tarvainen T, Aho H. Thompson hemiarthroplasty is superior to Ullevaal screws in treating displaced femoral neck fractures in patients over 75 years. A prospective randomized study with two-year follow-up. Ann Chir Gynaecol. 2001;90:225-8.

12. Görmeli G, Korkmaz MF, Görmeli CA, Adanaş C, Karataş T, Şimşek SA. Comparison of femur intertrochanteric fracture fixation with hemiarthroplasty and proximal femoral nail systems. Ulus Travma Acil Cerrahi Derg. 2015;21(6):503-508.

13. Harwin SF, Stern RE, Kulick RG. Primary BatemanLeinbach bipolar prosthetic replacement of the hip in the treatment of unstable intertrochanteric fractures in the elderly. Orthop. 1990;13:1131-1136.

14. Green S, Moore T, Proano F. Bipolar prosthetic replacement for the management of unstable intertrochanteric hip fractures in the elderly. Clin Orthop Relat. 1987;224:169-177.

15. R.K. Simmermacher, J. Ljungqvist, H. Bail, T. Hockertz, A.J. Vochteloo, U. Ochs, C.V. Werken, et al., The new proximal femoral nail antirotation (PFNA) in dailypractice: results of a multicentre clinical study. Injury. 2008;39;932-939.

16. E. Soucanye de Landevoisin, A. Bertani, P. Candoni, C. Charpail, E. Demortiere, Proximal femoral nail antirotation (PFN-ATM) fixation of extra-capsular proximal femoral fractures in the elderly: retrospective study in 102 patients. Orthop Traumatol Surg Res. 2012;98:288-295.

17. K. Sinno, M. Sakr, J. Girard, H. Khatib, The effectiveness of primary bipolar arthroplasty in treatment of unstable intertrochanteric fractures in elderly patients. $N \mathrm{Am} \mathrm{J} \mathrm{Med}$ Sci. 2010;2:561-568.
18. P. Sancheti KHS ancheti, A. Shyam, S. Patil, Q. Dhariwal, R. Joshi, Primary hemiarthroplasty for unstable osteoporotic intertrochanteric fractures in the elderly: a retrospective case series. Indian J Orthop. 2010;44:428434.

19. X. Chu, F. Liu, J. Huang, L. Chen, J. Li, P. Tong, Good short-term outcome of arthroplasty with Wagner SL implants for unstable intertrochanteric osteoporotic fractures. J Arthroplasty. 2014;29:605-608.

20. W.S. Choy, J.H. Ahn, J.H. Ko, B.S. Kam, D.H. Lee, Cementless bipolar hemiarthroplasty for unstable intertrochanteric fractures in elderly patients, Clin Orthop Surg. 2010(2):221-226.

21. Xiangping Luoa, Shengmao Heb, Dingshi Zengc, Lijun Linb, Qi Lib. Proximal femoral nail antirotation versus hemiarthroplasty in them treatment of senile intertrochanteric fractures: Case report. Int J Surg Case Reports. 2017;38:37-42.

22. Haentjens P, Casteleyn PP, De Boeck H, Handelberg F, Opdecam P. Treatment of unstable intertrochanteric and sub trochanteric fractures in elderly patients. Primary bipolar arthroplasty compared with internal fixation. $J$ Bone Joint Surg Am. 1989;71:1214-1225.

23. R. Siwach, H. Jain, R. Singh, K. Sangwa, Role of hemiarthroplasty in intertrochanteric fractures in elderly osteoporotic patients: a case series, Eur J Orthop Surg Traumatol. 2012;22:467-472.

24. N. Özkayın, G. Okc, u, K. Aktu glu, Intertrochanteric femur fractures in the elderly treated with either proximal femur nailing or hemiarthroplasty: a prospective randomised clinical study, Injury 46 (Suppl. 2) 2015:S38 .

How to cite this article: Saraf $\mathrm{H}$, Munot $\mathrm{S}$. Comparative study of PFN antirotation vs bipolar hemiarthroplasty in unstable senile intertrochanteric fractures. Indian J Orthop Surg. 2018;4(4):380-385. 